\title{
Ruptured Abdominal Aortic Aneurysm After Successful Transcatheter Aortic Valve Replacement- A Case Report and Review of Management
}

\author{
Daniella Ohnemus, Miriam Oren, Ezeldeen Abuelkasem and Michael S Trostler* \\ University of Pittsburgh Medical Center, Pittsburgh, PA, USA \\ *Corresponding author: Michael Trostler, Anesthesiology and Perioperative Medicine, University of Pittsburgh Medical Center, \\ Pittsburgh, PA, USA \\ Received date: 13 September, 2021 I Accepted date: 30 September, 2021 | Published date: 06 October, 2021 \\ Citation: Ohnemus D, Oren M, Abuelkasem E, Trostler MS (2021) Ruptured Abdominal Aortic Aneurysm After Successful Transcatheter \\ Aortic Valve Replacement- A Case Report and Review of Management. J Anaesth Anesth Drug 1(1). doi https://doi.org/ \\ $10.54289 / \mathrm{JAAD} 2100102$
}

Copyright: () 2021 Ohnemus D, et al. This is an open-access article distributed under the terms of the Creative Commons Attribution License, which permits unrestricted use, distribution, and reproduction in any medium, provided the original author and source are credited.

\section{Abstract:}

Background: Transcatheter aortic valve replacement is an important procedure with the aging US population presenting with more aortic stenosis and as many as $10 \%$ of these patients presenting with an abdominal aortic aneurysm at the time of screening. This procedure has also been shown to be as safe as open aortic valve replacement with lower risks of death, stroke, and rehospitalization.

Case Report: A 91-year-old female presents approximately one month after transcatheter aortic valve with an acute retroperitoneal rupture of a known abdominal aortic aneurysm. The patient was taken for an emergent endovascular aortic aneurysm repair and became suddenly unresponsive and apneic prior to any anesthetic drug administration, the patient was intubated, and rapid transfusion was started. The procedure was completed successfully with immediate improvement in the patient's vitals after deployment of the stent. The patient was recovering well, but five days later had a sudden pulseless electrical activity arrest and after appropriate but unsuccessful advanced cardiac life support was declared deceased.

Conclusion: While not standard of care, a simultaneous endovascular aneurysm repair during transcatheter aortic valve repair in select patients seems to be a safe procedure without increase in complications from either procedure completed separately. The aim of this manuscript is to review the recent success of simultaneous repair and to illustrate that this newer method may improve outcomes.

Keywords: Transcatheter Aortic Valve Replacement; Aortic Aneurysm Abdominal; Endovascular procedures; aortic rupture; aortic valve stenosis

Abbreviations: TAVR: Transcatheter aortic valve replacement, AS: Aortic Stenosis, AAA: Abdominal Aortic Aneurysm, rAAA: Ruptured Abdominal Aortic Aneurysm, EVAR: endovascular aneurysm repair, ADLs: activities of daily living, PRBCs: packed red blood cells, PEA: pulseless electrical activity, ACLS: Advanced cardiovascular life support, CKD: chronic kidney disease. 


\section{Background}

Transcatheter aortic valve replacement (TAVR) has become the standard of care for patients with severe aortic stenosis (AS) at intermediate or high risk for surgical intervention and has recently been shown to have a lower risk of death, stroke, and rehospitalization compared to open surgery in low-risk patients.[1] Patients who undergo TAVR often have comorbid vascular disease and up to $9.5 \%$ may have concomitant aortic aneurysm.[2]

While abdominal aortic aneurysm (AAA) is often asymptomatic, ruptured AAA (rAAA) carries a significant risk of mortality, two-thirds die before arriving at the hospital, and only $42 \%$ of those who receive treatment survive. Elective repair is typically offered when aneurysms reach a diameter of $5.5 \mathrm{~cm}$ or between $5.0-5.4 \mathrm{~cm}$ in women, above which risk of rupture is $5.3 \%$ per year.[3]

Traditionally, concomitant AS and AAA have been addressed sequentially. However, a growing number of case reports suggest that a simultaneous or "drive-by" endovascular aneurysm repair (EVAR) during TAVR for those with large or enlarging AAA is a feasible alternative to a sequential approach.

\section{Case Presentation}

A 91-year-old female presented to a local hospital with 12hours of severe, diffuse abdominal pain. History was significant for severe AS status post TAVR one-month prior, moderate mitral regurgitation, coronary artery disease, diastolic heart failure, atrial fibrillation with left bundle branch block and tachy-brady syndrome status post pacemaker, and a known AAA. She was found to have a rAAA and was transferred to the university hospital.

Prior to presentation, she was active and independent, recovering appropriately from the recent TAVR on apixaban, but no antiplatelet therapy. Her aortic stenosis was severe prior to TAVR with a valve area of $0.8 \mathrm{~cm}^{2}$ and a mean gradient of $34 \mathrm{mmHg}$ and was repaired with an Evolut Pro Plus $26 \mathrm{~mm}$ valve through a right common femoral cutdown. Her medical problems were stable and well managed, and she was able to perform all activities of daily living (ADLs) without assistance and had returned to a NYHA Class I status. Her AAA had been regularly monitored although prior imaging was not available. CT scan 6 months prior showed a diameter of 54 x $56 \mathrm{~mm}$ (approximately $25 \%$ increase from 43 x $44 \mathrm{~mm}$ several years prior). She was due for repeat CT scan in three months with plan for EVAR. She was now found to have a 58 x $59 \mathrm{~mm}$ aneurysm with infrarenal rupture and large left perinephric retroperitoneal hematoma. Imaging of the active extravasation from the rupture was not clear enough on any one image to include in this paper.

On arrival, the patient was transferred into the hybrid suite for emergent ruptured EVAR. The patient was hypertensive BP $200 / 93 \mathrm{mmHg}$, then after placement of a central line and without any sedating medications, the patient abruptly became apneic and unresponsive, and found to be hypotensive $84 / 47 \mathrm{mmHg}$ but responsive to vasopressors and massive transfusion of five units of uncrossed packed red blood cells (PRBCs), with only transient hypotension during the case, requiring $.08 \mathrm{mcg} / \mathrm{kg} / \mathrm{min}$ of norepinephrine as the maximum infusion dose, which was weaned off at the end of the case. Total operative time was 124 minutes, with 3285 $\mathrm{mL}$ of crystalloid and 5 units of PRBCs.

A successful EVAR was performed with a very small type 1a endoleak and hemodynamic improvement after graft deployment. Prior to transfer patient was acidotic $\mathrm{pH}-7.19$ and anemic with a hemoglobin of $6.9 \mathrm{~g} / \mathrm{dL}$, but a down trending Lactate, which increased after arrival in the ICU. It is likely that her sudden apnea and change in level of consciousness were due to worsening rupture or loss of containment in the retroperitoneum prior to the start of the procedure. Over the next 24 hours, the patient stabilized, and lactate normalized. On postoperative day five, the patient became acutely unresponsive and was found to be in pulseless electrical activity (PEA) arrest. The cardiac rhythm was noted to be sinus tachycardia with bigeminy. Advanced cardiovascular life support (ACLS) was initiated. The rhythm degenerated to ventricular fibrillation and the patient received multiple rounds of defibrillation as per ACLS algorithm. Unfortunately, after prolonged resuscitation efforts without return of spontaneous circulation and no clear cause for PEA, ACLS was stopped, and the patient was declared dead.

Although re-rupture leading to hypovolemia and PEA arrest was the most likely cause of death, per report there was no ultrasound performed at the time of the arrest, and the family 
declined post-mortem examination.

\section{Discussion}

This case represents a worst-case scenario for patients with concomitant AAA and AS. To date, there have been no trials investigating whether simultaneous TAVR and EVAR improves outcomes compared to a sequential approach. No guidelines exist to direct the sequence of management in patients who require both procedures. The patient was discharged on apixaban for anticoagulation therapy after TAVR, which may contribute to increased bleeding in the event of rupture, but with a new valve and her history of atrial fibrillation with left bundle branch block the risk of clot must be weighed against the risk of potential aortic aneurysm rupture. The timing of the EVAR should also be taken into consideration as a risk factor. In this case the new valve, history of arrythmia and plan for repair at 6 months led to the decision to anticoagulate.

Drive-by EVAR during TAVR was first described in the UK in 2012 [4] and in North America in 2014 [5]. Since then, multiple case reports and series have reiterated the feasibility of simultaneous repair with different configurations of endografts and valve devices. A 2019 case series of five patients reported no complications specific to performing both procedures simultaneously [6]. A number of published reports were well summarized by Rashid et al. (2017) [7] and Koutsias et al. (2020) [8]. Overall, simultaneous repair appears to be feasible, safe, and effective.

Simultaneous repair would be more cost effective and efficient, eliminating a second procedure, anesthetic, hospital stay and may improve the patient experience. Repeated vascular access is a known predictor of complications [9] which are among the most common adverse events related to EVAR and TAVR and contribute increase cost and length of stay $[10,11]$. Simultaneous repair of AS and AAA via the same access site could conceivably decrease the risks related to repeat arterial cannulation.

TAVR may increase the risk of aneurysm rupture, as relief of AS allows an increase in arterial pressure that can worsen strain on the aneurysm [12]. The TAVR procedure itself also presents increased risk for periprocedural complications in the setting of a narrowed, thrombus laden, or tortuous aortic lumen. Although the sequence in reported cases has varied depending on the hemodynamic and anatomic concerns unique to each patient, performing EVAR prior to TAVR, when possible, may decrease the risk of embolism, rupture, or dissection during advancement of the prosthetic valve or immediately following valve implantation.

AAA is typically well characterized prior to TAVR based on pre-procedure planning, making it an opportune time to coordinate care and address two sources of morbidity and mortality at once. However, it is important to note that a simultaneous approach necessitates multidisciplinary coordination which may not be feasible at all medical centers. Potential drawbacks may include increased operative time and contrast load. However, recorded procedure times and length of stay have been comparable to either procedure done separately [6]. Reported total amounts of contrast medium during simultaneous repair have also been within reasonable limits $(50-385 \mathrm{~mL})[\mathbf{6 , 8 , 1 3}]$. One case series reported no instances of acute kidney injury, even in those with chronic kidney disease (CKD) [6]. A separate patient with CKD actually demonstrated improved postoperative creatinine, perhaps due to hemodynamic changes following valve implantation [14].

Another concern is whether the potential benefits of a preventative procedure, such as EVAR, outweigh the immediate procedural risks in elderly patients with limited life expectancy. Aziz et al. (2016) [13] described a drive-by EVAR in a nonagenarian who tolerated the procedure without complication. However, two months later the patient enrolled in hospice after declining workup for new-onset confusion and lethargy [13]. This is a reminder that candidates must be carefully selected, with explicit discussions of goals of care prior to intervention. However, as life expectancy increases and endovascular techniques improve, the benefits may outweigh the risks in select patients.

\section{Conclusion:}

Our case highlights a potential consequence of delaying AAA repair in a patient with enlarging AAA undergoing TAVR. While data comparing outcomes of simultaneous versus sequential repair is lacking, a drive-by EVAR may have prevented aneurysm rupture in this patient. Simultaneous 
repair may also conceivably reduce costs, improve efficiency, and reduce risk of vascular complications for those who would otherwise need sequential TAVR and EVAR.

The number of patients with concomitant AS and AAA undergoing TAVR will continue increasing as TAVR becomes more prevalent. Further investigation is necessary to determine whether simultaneous repair should become standard of care in those with large or enlarging AAA.

This paper was considered exempt from IRB permission. The patient's next of kin consented for the use of all included information for the purposes of publication of this manuscript.

\section{References:}

1. Mack MJ, Leon MB, Thourani VH, Makkar R, Kodali SK, et al. (2019) Transcatheter Aortic-Valve Replacement with a Balloon-Expandable Valve in Low-Risk Patients. N Engl J Med. 380(18): 16951705 .

2. Kobayashi A, Lazkani M, Moualla S, Orazio A, Tasset M, et al. (2018) Impact of aortic aneurysms in trans-catheter aortic valve replacement: A single center experience. Indian Heart J. 70(3): S303-S308.

3. Parkinson F, Ferguson S, Lewis P, Williams IM, Twine CP. (2015) Rupture rates of untreated large abdominal aortic aneurysms in patients unfit for elective repair. J Vasc Surg. 61(6): 1606-1612.

4. Drury-Smith M, Garnham A, Khogali S. (2012) Critical aortic stenosis in a patient with a large saccular abdominal aortic aneurysm: Simultaneous Transcatheter Aortic Valve Implantation and Driveby Endovascular Aortic Aneurysm Repair. Catheter Cardiovasc Interv. 80(6): 1014-1018.

5. Aluko Y, Diehl L, Jacoby R, Chan B, Andrews S, et al. (2015) Simultaneous transcatheter aortic valve replacement and endovascular repair for critical aortic stenosis and large abdominal aortic aneurysm. Cardiovasc Revascularization Med. 16(4): 254-258.

6. Yammine H, Briggs CS, Rolle QV, Ballast JK, Frederick JR, et al. (2019) Simultaneous
Transcatheter Aortic Valve Replacement and Endovascular Aortic Aneurysm Repair. J Vasc Surg. 70(5): e133.

7. Rashid HN, McCormick LM, Gooley RP, Meredith IT. (2017) Simultaneous transcatheter aortic valve implantation and drive-by endovascular aortic aneurysm repair: a case of lotus valve retrieved and replaced due to an undersized valve after an endovascular aneurysm repair. Cardiovasc Interv Ther. 32(3): 299-303.

8. Koutsias S, Karaolanis GI, Papafaklis MI, Peroulis M, Tzimas P, et al. (2020) Simultaneous Transcatheter Aortic Valve Implantation and Infrarenal Aortic Aneurysm Repair for Severe Aortic Stenosis and Abdominal Aortic Aneurysm: Report of 2 Cases and Literature Review. Vasc Endovascular Surg. 54(6): 544-548.

9. Bhatty S, Cooke R, Shetty R, Jovin IS. (2011) Femoral vascular access-site complications in the cardiac catheterization laboratory: Diagnosis and management. Interv Cardiol. 3(4): 503-514.

10. Lange R, Bleiziffer S, Piazza N, Hutter A, TassaniPrell P, et al. (2011) Incidence and treatment of procedural cardiovascular complications associated with trans-arterial and trans-apical interventional aortic valve implantation in 412 consecutive patients. Eur J Cardio-thoracic Surg. 40(5): 1105-1113.

11. Mwipatayi BP, Picardo A, Masilonyane-Jones TV, Larbalestier R, Thomas S, et al. (2013) Incidence and prognosis of vascular complications after transcatheter aortic valve implantation. J Vasc Surg. 58(4): 1028-1036.e1.

12. Gotzmann M, Hehen T, Germing A, Lindstaedt M, Yazar A, et al. (2010) Short-term effects of transcatheter aortic valve implantation on neurohormonal activation, quality of life and 6minute walk test in severe and symptomatic aortic stenosis. Heart. 96(14): 1102-1106. 
13. Aziz F, Kozak M, Pae WE. (2016) Combined Transfemoral Aortic Valve Replacement and Endovascular Repair of Abdominal Aortic Aneurysm in Nonagenerians is Feasible. Clin Surg. 1(1): 1153.
14. Orejola WC, Elmann EM, Wilderman MJ, Cocke TP, Vaidya P, et al. (2016) Combined Transcatheter Aortic Valve Replacement and Endovascular Aortic Repair: A Transfemoral Approach Dilemma. J Surg. 4(1): 3 